DOI: $10.3901 / J M E .2020 .22 .247$

\title{
地铁车辆车轮偏磨原因分析与对策研究 ${ }^{*}$
}

\author{
肖国放 ${ }^{1}$ 陶功权 ${ }^{1}$ 刘孟奇 ${ }^{1}$ 任德祥 ${ }^{1}$ 温泽峰 ${ }^{1}$ 周小江 ${ }^{2}$ 陆文教 ${ }^{3}$ \\ (1. 西南交通大学牵引动力国家重点实验室 成都 610031; \\ 2. 中车青岛四方机车车辆股份有限公司 青岛 266111; \\ 3. 广州地铁集团有限公司 广州 530330)
}

\begin{abstract}
摘要: 轮对偏磨是铁道车辆常见的车轮磨耗形式。对国内某地铁线路的车轮磨耗进行测试分析, 发现该线路车辆存在严重的 车轮偏磨现象, 左侧车轮以轮缘磨耗为主, 右侧车轮以踏面磨耗为主。该地铁线路小半径曲线多, 且左、右曲线分布严重不 均, 以及车辆不掉头运行是造成车轮偏磨的主要原因。基于 UM 动力学软件建立车轮磨耗预测模型, 利用地铁车轮磨耗测试 结果对磨耗预测模型进行验证, 根据数值仿真结果提出轮对偏磨的解决措施。仿真结果表明, 车辆掉头行驶能明显减缓车轮 的偏磨现象, 最佳掉头运行里程数为 $2 \times 10^{4} \sim 4 \times 10^{4} \mathrm{~km}$ 。小半径曲线占比对车轮磨耗影响较大, 左、右曲线百分比差值小于 $3 \%$ 时可不采取掉头措施。
\end{abstract}

关键词: 地铁车辆; 车轮磨耗; 偏磨; 数值仿真; 小半径曲线

中图分类号: U211

\section{Causations and Countermeasures of Wheel Partial Wear of Metro Vehicles}

\author{
XIAO Guofang ${ }^{1} \quad$ TAO Gongquan $^{1} \quad$ LIU Mengqi $^{1} \quad$ REN Dexiang $^{1} \quad$ WEN Zefeng $^{1}$ \\ ZHOU Xiaojiang $^{2}$ LU Wenjiao $^{3}$
}

(1. State Key Laboratory of Traction Power, Southwest Jiaotong University, Chengdu 610031;

2. CRRC Qingdao Sifang Co., Ltd., Qingdao 266111;

3. Guangzhou Metro Group Co., Ltd., Guangzhou 530330)

\begin{abstract}
The partial wear of wheelset is a common form of wheel wear for railway vehicles. Through a wheel wear investigation of a certain metro line in China, a significant partial wear phenomenon is observed. The left wheels are mainly worn on the flange, while the right wheels are mainly worn on the tread. The principle causes of the wheel partial wear are that the metro line possesses many small radius curves, the distribution of the left and right curves is severely uneven, and the vehicles don't reverse periodically. In order to mitigate the wheel partial wear, the wheel wear prediction model is established based on the UM dynamics software, which is verified by the metro wheel wear measurement results. According to the numerical simulation results, the solutions of the wheel partial wear are proposed. The vehicle periodical reversion can significantly mitigate the wheel partial wear, and the optimal reversion mileage is $2 \times 10^{4}-4 \times 10^{4} \mathrm{~km}$. The proportion of the small radius curve has a great influence on the wheel wear. It does not need to take the measure of periodical reversion when the percentage difference between the left and right curves is less than $3 \%$.
\end{abstract}

Key words: metro vehicle; wheel wear; partial wear; numerical simulation; small radius curves

\section{0 前言}

随着城市经济的繁荣，轨道交通行业迅猛崛起 和发展, 地铁作为重要的交通工具给人们的出行带 来了非常大的便利。与此同时, 城市人口的增长给 城轨交通运输造成的压力也越来越大。受到城市规

* 四川省科技计划资助项目(2019YFH0053，20YYJC3766)。20191201 收 到初稿, 20200501 收到修改稿
划建设和发展的限制，地铁线路不得不设置较多的 小半径曲线。地铁列车在小半径曲线上运行时轮轨 相互作用更为剧烈, 轮轨间的蠕滑力和蝡滑率要显 著大于大半径曲线和直线段, 由此导致的轮轨磨耗 问题也更为突出，轮对非对称磨耗就是其中典型的 磨耗问题。轮对非对称磨耗将对车辆运行的稳定性、 平稳性和舒适性产生极大的影响, 甚至会威胁列车 的行车安全。此外, 轮对非对称磨耗也会对车轮的 维护带来不利影响。因此, 研究车轮偏磨产生的原 因并提出相应的对策就显得十分重要。 
据文献报道, 我国福州、哈尔滨、深圳、重庆 等多条地铁和高铁线路车轮轮缘或踏面都出现不同 程度的异常磨耗 ${ }^{[1-4]}$, 其中轮缘偏磨或踏面偏磨现象 尤为严重, 文献[1-4]以车轮磨耗现场测试为基础, 结合实际运营维护经验, 提出了一些轮对异常磨耗 预防和减缓措施。杨斌等 ${ }^{[5]}$ 基于实验室获得的 CL60 车轮材料 $T \gamma / A$-磨损率曲线建立车轮踏面磨损模型, 对车轮踏面的磨耗规律进行仿真分析, 研究表明相 同工况使用该模型获得的仿真结果比采用 Archard 磨损系数仿真得到的结果要小, 其结果具有更好的 精度。周素霞等 ${ }^{[6]}$ 结合实测轮轨磨耗型面, 利用 ANSYS 建立曲线段轮轨三维弹塑性接触有限元模 型, 分析了不同磨耗状态下轮轨接触应力分布, 发 现随钢轨侧磨增加, 轮轨接触状态由一点接触变为 两点接触, 轨距角易出现较大的接触应力, 加速钢 轨磨耗与疲劳伤损的产生。LI 等 ${ }^{[7-8]}$ 介绍了车轮磨耗 的预测方法, 详细分析了地铁车轮踏面异常磨耗产 生的原因, 认为闸瓦制动是其中的关键因素, 并且 研究了地铁车辆车轮轮缘磨耗的成因, 采用数值方 法对异常磨耗车轮和新钢轨之间的相互作用进行了 详细地论述。李艳等 ${ }^{[9-10]}$ 研究了车轮踏面外形和轮 径差对车辆动力学性能的影响, 指出车轮踏面磨耗 导致车轮外形改变, 加剧轮径的改变, 产生非对称 现象, 将严重影响车辆的平稳性, 甚至还会威胁到 列车的行车安全。于春广等 ${ }^{[11]}$ 结合现场试验, 分析 了某条地铁线路的磨耗特征, 阐述了造成轮缘偏磨 的原因, 从轮轨关系的角度出发, 研究了一系纵向 刚度及摩擦系数等对车轮磨耗的影响, 提出了相应 的车轮磨耗减缓措施。FRÖHLING ${ }^{[12]}$ 基于南非铁路 网公司煤炭出口线的现场测试数据, 研究了车轮踏 面偏磨产生的原因及其影响, 论述了偏磨对于车轮、 转向架、钢轨、道岔等结构的危害。池茂儒等 ${ }^{[13]}$ 通 过研究有轮径差的转向架, 推断出轮径差会改变轮 对的对中平衡位置, 导致轮轨接触关系改变, 进而 影响车辆系统的稳定性, 并将其同时划分为易稳定 区、欠稳定区和亚稳定区，踏面偏磨容易发生在欠 稳定区，轮缘偏磨易发生在亚稳定区。黄照伟等 ${ }^{[14]}$ 对某高速列车车轮踏面磨耗进行了长期跟踪测试, 发现存在较为明显的轮对偏磨现象。通过仿真计算 分析了偏磨与踏面等效雉度之间的变化关系, 发现 负雉度现象导致偏磨速率加快, 且同相偏磨对车辆 运行平稳性及临界速度的影响较反相偏磨严重。

KARTTUNEN 等 ${ }^{[15]}$ 评估了磨耗车轮踏面对轮轨接 触的影响, 指出了现阶段存在的问题。目前铁道车 辆车轮的维护标准主要针对磨耗深度、轮缘厚度、 轮缘梯度、轮缘高度等指标, 这些几何特征量便于
测量, 但无法详尽地描述出踏面磨耗状态及对轮轨 关系恶化的影响, 同时给出了一种参数化的磨耗车 轮型面操作步骤, 具体详细地分析了其可行性和完 善性。

目前针对车轮偏磨的研究大多集中在定性分析 上, 主要研究其对车辆系统动力学的影响, 缺少对 车辆车轮偏磨成因以及解决方法的深入探索。本文 针对某条地铁线的车轮偏磨现象进行研究。通过现 场试验掌握轮缘、踏面磨耗规律，同时全面调查该 线路特征以及车辆运营信息; 建立车辆-轨道动力学 仿真模型、轮轨滚动接触模型、材料摩擦磨损模型、 型面平滑与更新和轨道线路模型为一体的车轮磨耗 预测模型，其中轮轨滚动接触计算采用非椭圆滚动 接触算法, 轮轨磨耗之后接触斑内曲率不再为常数, 车轮与钢轨将发生非椭圆接触, 该算法能够更准确 的模拟轮轨接触状态。对车轮磨耗预测结果从磨耗 深度和磨耗后车轮踏面外形两个方面进行验证, 研 究车轮偏磨原因, 针对偏磨问题提出解决方案, 并 定量地分析解决方案的有效性和可行性。

\section{1 车轮磨耗测试及分析}

对国内某条地铁线路的车轮磨耗进行了大量测 试, 获得了 13 列地铁车辆(本文对其编号 $\mathrm{T} 1$ 至 $\mathrm{T} 13$ ) 所有车轮的磨耗状态, 测试时车轮均未进行钫修。 车辆在该线路为不掉头运行, 车轮磨耗测试时车辆 的运行里程从 $5 \times 10^{4} \mathrm{~km}$ 到 $15 \times 10^{4} \mathrm{~km}$ 不等。测量所 得某轮对左右侧车轮的磨耗状态如图 1 所示。由于 测试车辆运行线路复杂, 小半径曲线众多, 导致磨 耗区域较宽，磨耗分布在-45 45 mm 范围内。左右 侧车轮磨耗位置存在较大差异, 左侧车轮轮缘磨耗 非常明显， $-33 \sim-43 \mathrm{~mm}$ 范围内的磨耗明显大于右 侧车轮; 右侧车轮踏面区域( $-20 \sim 20 \mathrm{~mm}$ 范围内)磨 耗比左侧车轮至少高出 $1 \mathrm{~mm}$ 。该线路车辆轮对出现 典型的偏磨现象, 且这种磨耗状态普遍存在于所有 测试车辆中。

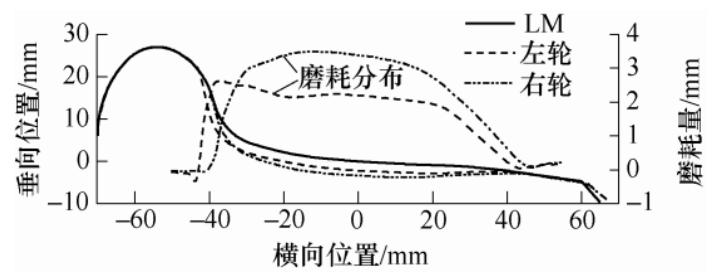

图 1 同一轮对左右车轮典型磨耗形式

对所测试的 13 列车车轮磨耗进行统计分析。面 向 1 车所有左侧车轮定义为左轮, 右侧车轮定义为 右轮。图 2 为左右侧车轮轮缘平均磨耗速率和踏面 
平均磨耗速率统计结果。所有测试的车辆均出现偏 磨现象, 左轮轮缘磨耗速率大于右侧, 左右侧车轮 轮缘磨耗速率之比约为 $1.67: 1$; 右轮踏面磨耗速率 大于左轮, 右轮和左轮踏面磨耗速率之比约为 $1.42: 1$ 。车轮踏面产生偏磨后必然会导致同一轮对 轮径差增大, 而滚动圆半径差是车辆动力学性能主 要评价指标, 决定了车辆直线运动的平稳性、稳定 性及曲线通过性能。在无偏磨情况下, 车辆直线行 驶时轮对左右晃动具有相同的几率, 转向架在轨道 中心附近振动。当出现偏磨情况时, 轮对将会向轮 径较小一侧偏移, 导致转向架有一定的摇头运动, 从而大大降低车辆蛇行失稳临界速度。车辆曲线通 过时, 离心力导致轮对向曲线外轨偏靠, 使外轨车 轮滚动圆半径大于内轨车轮滚动圆半径, 外轨将会 滚过较长弧线, 引导车辆通过曲线。偏磨情况下轮 径差较大, 有利于单侧曲线通过, 对反向曲线通过 时影响较大。同时, 左、右侧车轮轮缘偏磨对车轮 锁修造成不利影响, 轮缘每磨耗 $1 \mathrm{~mm}$, 要使其恢复 标准轮缘厚度, 踏面就要锭掉 $3 \sim 4 \mathrm{~mm}$, 由此造成 两侧车轮锭修量严重失衡, 不利于车轮维护, 且频 繁镢修将极大缩短车轮使用寿命。

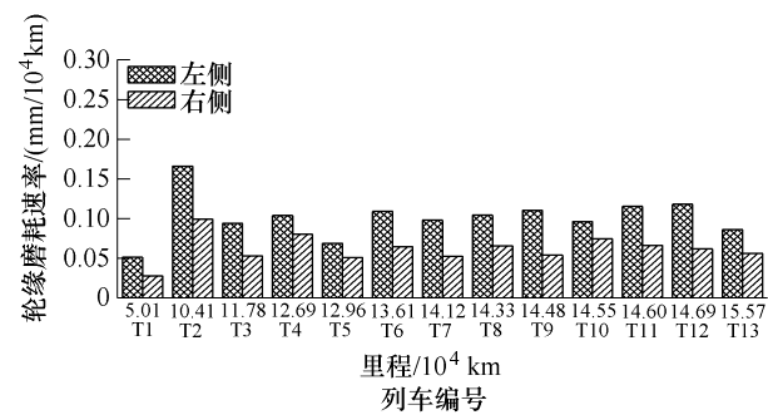

(a) 轮缘磨耗量统计

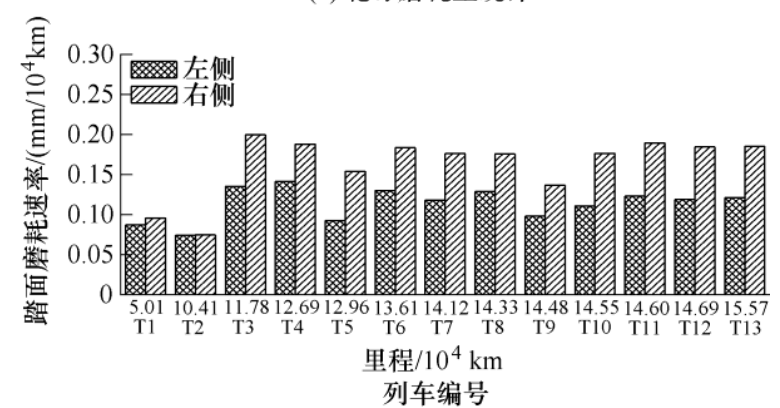

(b) 踏面磨耗量统计

图 2 车轮磨耗统计

\section{2 车轮磨耗预测模型及验证}

图 3 给出车轮磨耗预测流程, 由以下几个模块 组成: 车辆-轨道动力学仿真模型、轮轨接触局部分 析、车轮磨耗量计算、型面平滑与更新。

车轮磨耗预测具体过程如下: 首先进行车辆-

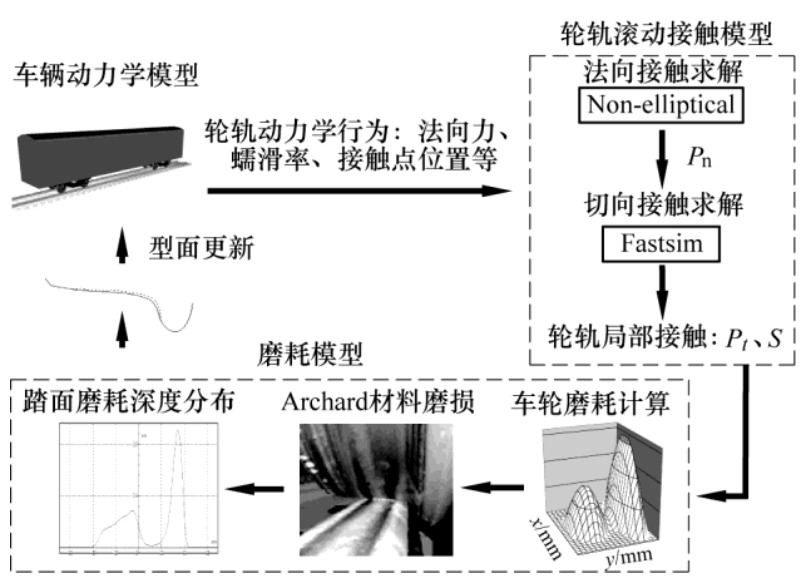

图 3 车轮磨耗预测流程图

轨道动力学仿真计算得到轮轨动力学行为, 包括法 向力 $P$ 、纵向蠕滑率 $v_{x}$ 、横向蠕滑率 $v_{y}$ 、自旋蠕滑 率 $\phi$ 、轮轨接触点位置等参量, 用于局部接触分析; 利用轮轨滚动接触模型对接触斑形状、蠕滑力分布 以及黏滑区分布进行计算; 采用 Archard 材料摩擦 磨损模型进行磨损量计算, 得到接触斑内磨耗分布, 并将仿真得到的磨耗进行叠加, 得到车轮型面的磨 耗分布; 最后将计算得到的磨耗车轮踏面外形返回 到车辆-轨道动力学仿真模型中进行下一次计算, 如 此迭代直至满足要求。接下来对车轮磨耗预测模型 中的各子模型进行介绍。

\section{1 车辆动力学模型}

地铁车辆是由许多部件组成的复杂多体系统, 各个部件之间相互作用, 形成一个整体。依据本次 研究的地铁车辆结构特性和动力学参数, 在 UM 多 体动力学仿真软件中建立了车辆动力学模型, 模型 包括 1 个车体、2 个构架和 4 条轮对。转向架采用 两系悬挂, 其中一系悬挂为叠层橡胶弹簧, 二系悬 挂包括大挠度高柔性的空气弹簧、单侧横向减振器、 横向止挡和甡引拉杆。横向止挡和二系横向减振器 均考虑其非线性特性。考虑到本文的研究重点为车 轮磨耗, 为突出重点方便计算, 故在建立动力学模 型时做以下考虑: (1) 将主要的实体部件如车体、构 架、轮对等结构视为理想不变形的刚体; (2) 不考虑 钢轨弹性变形; (3) 车轮型面为 LM 型踏面, 钢轨为 $60 \mathrm{~kg} / \mathrm{m}$ 型面。仿真时车辆考虑为重车状态, 部分车 辆参数如表 1 所示。

\section{2 轮轨滚动接触模型}

磨耗计算中, 材料的磨耗量主要取决于接触斑 内粘滑区分布和蠕滑力大小。轮轨滚动接触理 论 ${ }^{[16-17]}$ 是决定车轮磨耗预测结果的关键因素。赫兹 接触受到弹性半空间假设的限制, 要求接触斑的几 何尺寸远小于接触处的曲率半径, 并且假设接触斑 内曲率为常数。车轮磨耗之后接触斑内的曲率基本 


\section{表 1 部分车辆参数}

\begin{tabular}{cc}
\hline 名称 & AW3 重车 \\
\hline 轮对质量 $/ \mathrm{kg}$ & 1290 \\
构架质量 $/ \mathrm{kg}$ & 2106 \\
车体质量 $/ \mathrm{kg}$ & 39494 \\
一系纵向刚度 $/(\mathrm{MN} / \mathrm{m})$ & 3.3 \\
一系横向刚度 $/(\mathrm{MN} / \mathrm{m})$ & 2.45 \\
一系垂向刚度 $/(\mathrm{MN} / \mathrm{m})$ & 0.7 \\
空气弹簧纵向刚度 $/(\mathrm{MN} / \mathrm{m})$ & 0.12 \\
空气弹簧横向刚度 $/(\mathrm{MN} / \mathrm{m})$ & 0.12 \\
空气弹簧垂向刚度 $/(\mathrm{MN} / \mathrm{m})$ & 0.25 \\
车辆定距 $/ \mathrm{m}$ & 12.6 \\
轴距 $/ \mathrm{m}$ & 2.2 \\
\hline
\end{tabular}

不再为常数, 为了更好地模拟接触状态, 提高接触 计算的精度, 采用了非栯圆滚动接触算法 ${ }^{[18]}$ 。考虑 到轮轨材料属性上的相似性, 将轮轨间的接触分成 法向与切向接触分别计算。在计算非椭圆接触区域 切向应力的分布时, 采用了适用于非椭圆接触的 FASTSIM 修正算法, 用等效椭圆的方式, 即将非 椭圆接触斑的区域等效于椭圆区域来进行计算。在 滚动方向上椭圆的半轴长被认为与非椭圆接触斑 的最大半长相等。FASTSIM 算法是不限于椭圆接 触的一般方法, 对非椭圆形接触斑也可得到较好的 计算结果。

\subsection{Archard 材料摩擦磨损模型}

本文采用 Archard 磨耗模型 ${ }^{[19]}$ 进行车轮磨耗计 算。在该模型中材料的磨耗体积与接触斑内的法向 力和相对滑动距离的乘积成正比, 与材料硬度成反 比, 关系如式(1)所示

$$
V=k_{v} \frac{F d}{\rho H}
$$

式中, $V$ 表示材料磨耗体积; $d$ 表示相对滑动距离; $F$ 表示轮轨法向力; $H$ 表示两接触体中硬度较低材 料的布氏硬度大小; $k_{v}$ 表示磨耗因数, 与蠕滑速度 和法向压力相关。

在利用此模型计算轮轨磨耗时, 需要根据滑动 速度和接触压力确定磨耗因数 $k_{v}$ 的大小。图 4 为 Archard 磨耗模型中磨耗因数的取值范围。该磨耗因 数通过试验获得, 由图可以看出 Archard 磨耗图将 磨耗分为三个区域, 即轻微磨耗区、严重磨耗区和 灾难性磨耗区。UM 软件中使用的 Archard 磨耗模 型中磨耗因数与图 4 有些区别, 但也是相通的。UM 将式(1)中 $F d$ 定义为摩擦功 $A, k_{v} / \rho H$ 定义为新的磨 耗因数 $k_{w}$, 则磨耗体积如式(2)所示

$$
V=k_{w} A
$$

则有 $k_{\downarrow} / \rho H=k_{w}$, 对于车轮材料, $\rho=7800 \mathrm{~kg} / \mathrm{m}^{3}$, $H=280 \mathrm{MPa}$, 当 $k_{v}$ 取值范围是 $(1 \sim 400) \times 10^{-4}$ 时, $k_{w}$
取值范围是 $(0.05 \sim 18.32) \times 10^{-15}$, 在 $\mathrm{UM}$ 软件 Archard 磨耗模型中, 可取 $k_{w}$ 为定值, 为使得仿真 结果更加符合地铁车辆车轮磨耗形状, 对取值做适 当调整, 结果证明, 当 $k_{w}=0.15 \times 10^{-15}$, 即 $k_{v}=3.27 \times$ $10^{-4}$ 时, 所有磨耗因素落于 Archard 磨耗模型( 1 $10) \times 10^{-4}$ 区域内, 车轮磨耗量大小与磨耗区域更加 符合实测结果。

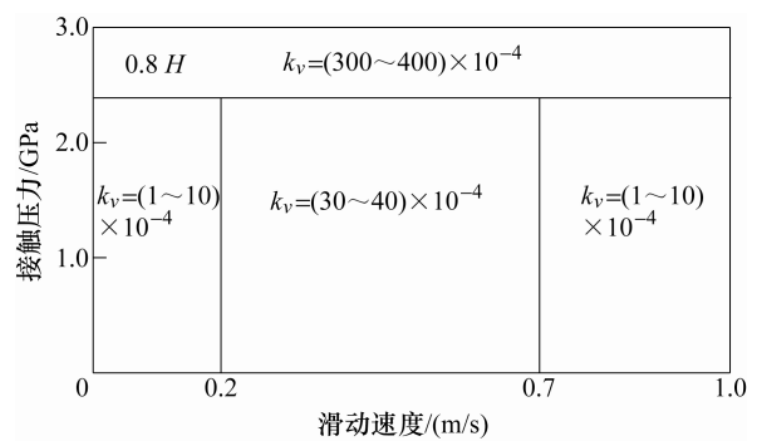

图 4 Archard 磨耗因数

\section{4 型面平滑与更新}

车辆运行时车轮磨耗是连续变化的过程。在仿 真计算中首先进行车辆动力学计算, 单次迭代计算 时, 型面保持不变, 即磨耗的计算定义成线性变化 的过程。每次迭代计算结束后，由磨耗模型可得磨 耗速率、磨耗量和迭代步长对应的信息, 同时型面 符合更新机制将会自动更新，计算后的型面会作为 新型面进入下一次迭代计算步中。

本文采用一种自适应步长算法, 该算法的特点 是能够根据磨耗速率不断调整迭代步长的大小来避 免定步长带来的计算结果偏大或偏小的问题，以提 高计算准确度。车辆运行距离和车轮磨耗深度是用 来限制磨耗步长的两种方法。相关研究表明, 以运 行距离为更新策略时可能会出现单次迭代磨耗强度 较大而使磨耗量偏大的问题。本文以车轮最大磨耗 深度 $0.1 \mathrm{~mm}$ 作为型面更新依据, 以控制车轮型面的 变化。磨耗计算后对磨耗分布进行三次样条插值平 滑, 之后将磨耗分布叠加到上一次的车轮型面, 同 样进行三次样条插值平滑。

\section{5 轨道线路模型}

车轮磨耗对轨道线路的变化非常敏感。所调查 线路总长超过 $24 \mathrm{~km}$, 线路较复杂。为避免在真实 轨道上进行过长时间的模拟，又能保证计算结果的 准确性，本文使用一种合理化的轨道统计方法来简 化整个仿真计算工作，即用短曲线轨道上的等效模 拟代替整个轨道上的模拟, 每一组曲线均设置对应 半径和超高, 具体工作如下。

（1）基于实际轨道调查，确定了一组合适的曲 线半径间隔, $700 \mathrm{~m}$ 以下半径以 $100 \mathrm{~m}$ 作为采样间 
隔, $700 \mathrm{~m}$ 以上半径则以 $200 \mathrm{~m}$ 为采样间隔。

(2) 对于每一组短曲线, 使用曲线长度作为加 权因子, 所有曲线半径的加权平均值作为该组短曲 线的代表半径 $R$ 。该组曲线中出现频次最高的超高 值作为在该半径下的代表超高 $h$ 。

(3) 以曲线匀速运行长度作为加权因子, 所有 曲线运行速度的加权平均值作为每组短曲线中车辆 运行速度的代表值。

(4) 左曲线/右曲线比例为该组短曲线中左曲 线/右曲线长度与线路总长的比值。

由于每组短曲线长度均不相同, 其对车轮磨耗 的贡献量按照每组曲线长度占线路总长的比例计
算, $d_{i}$ 为线路 $i$ 的权重

$$
\begin{gathered}
d_{i}=\frac{s_{i}}{s} \\
\sum_{i=1}^{M} d_{i}=1
\end{gathered}
$$

式中, $s_{i}$ 为短曲线 $i$ 的长度; $s$ 为线路总长度; $M$ 为 短曲线总条数。

本文的线路统计未考虑轨道上下坡道、汼引/ 制动、道岔及进出停车库 (场) 线路等的影响, 结果 如表 2 所示, 由 12 条曲线 (包含左/右曲线) 和 1 条 直线轨道组成, 实际线路无 $\mathrm{R} 500 \sim \mathrm{R} 600$ 半径范围 内曲线。

\begin{tabular}{|c|c|c|c|c|c|c|c|}
\hline 半径范围/m & 曲线半径/m & 曲线长度/m & 超高/mm & 速度/ $(\mathrm{km} / \mathrm{h})$ & 左曲线(\%) & 右曲线(\%) & 累积(\%) \\
\hline $0 \sim 400$ & 350 & 2945 & 115 & 61 & 7.07 & 4.73 & 11.8 \\
\hline $400 \sim 500$ & 450 & 4720 & 105 & 62 & 11 & 6.73 & 29.5 \\
\hline $500 \sim 600$ & - & - & - & - & - & - & - \\
\hline $600 \sim 700$ & 650 & 456 & 90 & 66 & 1.22 & 0.62 & 31.3 \\
\hline $700 \sim 900$ & 800 & 359 & 75 & 67 & 0.56 & 0.89 & 32.8 \\
\hline $900 \sim 1100$ & 1000 & 137 & 65 & 69 & 0.55 & 0 & 33.3 \\
\hline $1100 \sim 1300$ & 1200 & 453 & 40 & 68 & 0.89 & 0.94 & 35.2 \\
\hline $1300 \sim 1600$ & 1500 & 375 & 35 & 71 & 0.75 & 0.77 & 36.7 \\
\hline 直线 & - & - & - & 71 & \multicolumn{2}{|c|}{63.3} & 100 \\
\hline
\end{tabular}

表 2 线路统计参数

\section{6 车轮磨耗预测模型验证及分析}

在计算过程中, 本文有如下约定: (1) 本文左轮 位于外轨的曲线定义为左曲线; (2) 车辆不掉头往返 运行, 总里程设置为 $20 \times 10^{4} \mathrm{~km}$; (3) 计算中忽略钢 轨型面的磨耗; (4) 考虑轨道线路存在轨侧润滑装 置, 将 R400 以下半径曲线外轨 (高轨) 侧轮缘与钢 轨接触区域摩擦因数调低至 $0.2 \sim 0.3$, 其余每条曲 线或直线轮轨摩擦因数为 0.4 。基于以上假设, 车辆 $1 、 4$ 位轮对磨耗相同, $2 、 3$ 位轮对磨耗相同, 后文 只给出了第 1 和第 2 位轮对的车轮磨耗仿真结果。

图 5a、5b 和图 $6 a 、 6 b$ 分别给出了第 1 和第 2 位轮对左右侧车轮型面在不同运行里程情况下的磨 耗分布和车轮外形。由图可以看出, 车轮磨耗分布 出现在车轮型面横向位置- $42 \sim 40 \mathrm{~mm}$ 范围内, 1 位 轮对与 2 位轮对磨耗类似, 车轮踏面的磨耗深度随 运行里程的增加基本呈线性变化, 相同运行里程下 第 1 位轮对磨耗深度稍大于第 2 位轮对。左侧车轮 最大磨耗深度出现在横向位置 $-42 \sim-35 \mathrm{~mm}$ 范围 内, 轮缘磨耗较为严重, 踏面中部磨耗较轮缘轻; 右侧车轮磨耗主要发生在车轮名义滚动圆 $\pm 25 \mathrm{~mm}$ 范围内, 踏面中部磨耗深度比左侧车轮高出 $1 \mathrm{~mm}$
左右, 轮缘磨耗较左侧轻, 呈现出明显的车轮偏磨 现象。磨耗模型预测结果与车轮磨耗测试结果基本 一致, 仿真得到的车轮踏面外形与车轮实际磨耗状 态较为接近, 车轮偏磨规律吻合较好, 验证了车轮 磨耗预测模型的准确性。

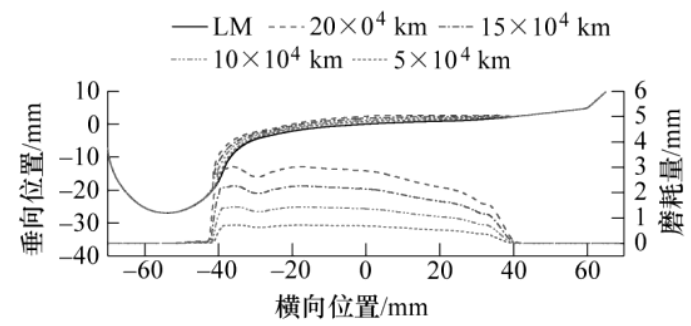

(a) 第1位轮对左轮

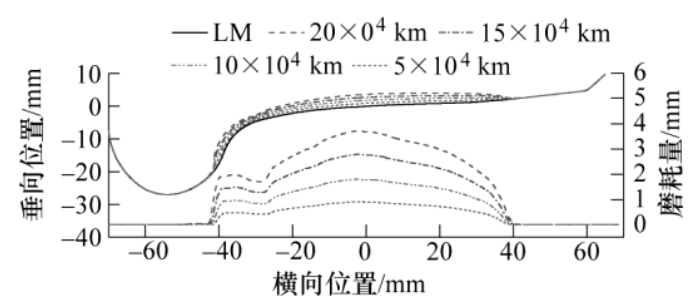

(b) 第1位轮对右轮

图 5 第 1 位车轮型面磨耗分布及外形情况

结合车轮磨耗试验测试结果及数值仿真结果, 对车轮偏磨问题进行研究。从线路方面分析, 小半 


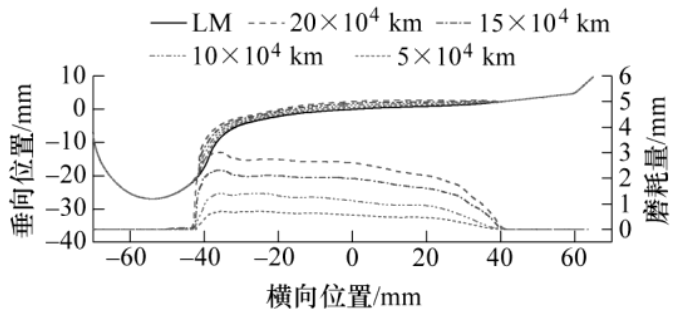

(a) 第2位轮对左轮

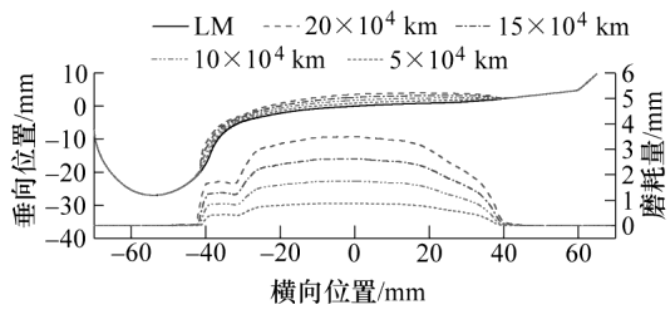

(b) 第2位轮对右轮

图 6 第 2 位车轮型面磨耗分布及外形情况

径曲线在所有曲线段中占据绝对比例, 且小半径曲 线的分布存在明显的不对称现象, 尤其在对轮缘磨 耗贡献较大的半径小于 $500 \mathrm{~m}$ 的曲线中, 左曲线所 占的比例几乎为右曲线的 2 倍。在车辆通过小半径 左曲线时, 由于发生轮缘贴靠现象, 导致轮缘与钢 轨剧烈摩擦, 使得左轮轮缘磨耗较右轮大, 这是造 成轮缘偏磨的主要原因; 整条线路左右曲线不对称 分布, 使得左右侧踏面磨耗位置不同, 造成踏面偏 磨。从运营方面分析, 车辆采取不掉头往返运行方 式, 使得车辆左侧车轮轮缘与钢轨作用时间比右侧 长, 踏面与钢轨接触位置几乎不发生改变, 偏磨问 题得不到缓解, 随车辆运行里程的增加偏磨现象更 加严重, 对行车安全造成隐患。

\section{3 车轮偏磨解决对策}

\section{1 掉头对车轮磨耗的影响}

受城市规划建设的限制, 地铁线路条件无法改 变, 小半径曲线占比高及左右曲线比例不均等因素 依然存在。从车辆运营方面着手, 定期掉头是缓解 车轮偏磨问题的有效措施。为研究掉头对车轮磨耗 的影响, 确定合适的掉头里程, 由车轮磨耗预测模 型及轨道线路模型, 继续进行数值仿真计算。车辆 运行总里程 $20 \times 10^{4} \mathrm{~km}$ 不变, 设置四种工况, 使车 辆掉头行驶里程数分别为 $10 \times 10^{4} \mathrm{~km} 、 5 \times 10^{4} \mathrm{~km}$ 、 $4 \times 10^{4} \mathrm{~km}$ 和 $2 \times 10^{4} \mathrm{~km}$, 分析每种工况下左右侧车 轮的磨耗情况。图 7 和图 8 给出不同掉头里程下 左右侧车轮型面磨耗分布和外形, 同时给出车辆每 进行一次掉头时左右侧车轮型面磨耗情况。篇幅所 限, 本文仅列出 $10 \times 10^{4} \mathrm{~km}$ 及 $2 \times 10^{4} \mathrm{~km}$ 掉头工况 下的结果。

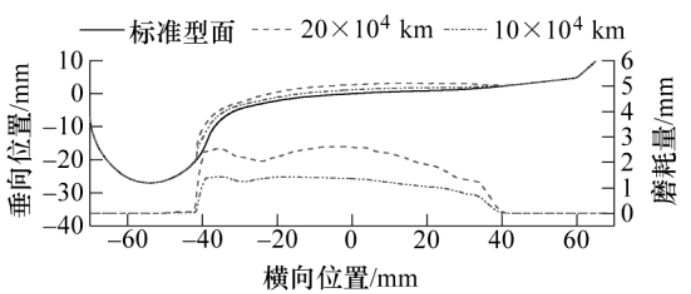

(a) 左侧车轮

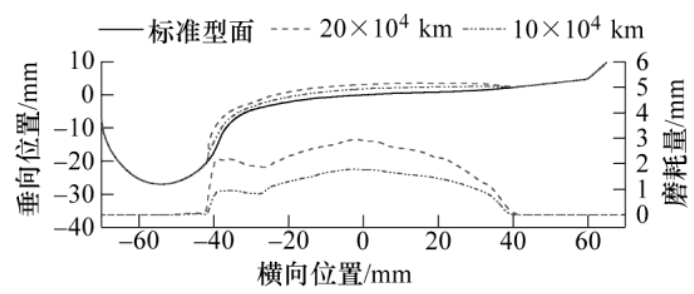

(b) 右侧车轮

图 $710 \times 10^{4} \mathrm{~km}$ 掉头车轮型面磨耗分布及外形情况

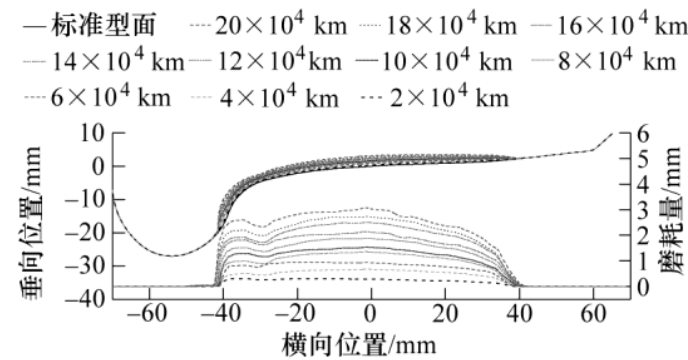

(a) 左侧车轮

一标准型面 $\quad-20 \times 10^{4} \mathrm{~km}-18 \times 10^{4} \mathrm{~km}-16 \times 10^{4} \mathrm{~km}$ $-14 \times 10^{4} \mathrm{~km}-12 \times 10^{4} \mathrm{~km}-10 \times 10^{4} \mathrm{~km}-8 \times 10^{4} \mathrm{~km}$ $-6 \times 10^{4} \mathrm{~km} \quad 4 \times 10^{4} \mathrm{~km} \quad \cdots 2 \times 10^{4} \mathrm{~km}$

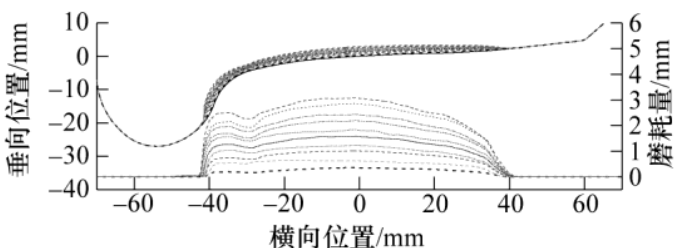

(b) 右侧车轮

图 $82 \times 10^{4} \mathrm{~km}$ 掉头车轮型面磨耗分布及外形情况

仿真结果表明，车辆定期掉头行驶能够有效缓 解左右侧车轮偏磨现象。由车轮型面磨耗变化可知, 车辆每进行一次掉头行驶后, 由于左右侧车轮对应 的左右曲线比例的变化, 车轮磨耗情况相应改变, 随着车辆的不断掉头行驶, 左右侧车轮磨耗较为接 近, 车轮外形趋于一致。

图 9 给出不同掉头里程下, 左右侧车轮磨耗差 随运行里程的变化情况, 可以看出, 随运行里程的 增加, 各个工况下的轮缘磨耗差与踏面磨耗差均有 明显下降，特别是第一次掉头之后，磨耗差迅速下 降, 随后稳步减小。这是由于经过掉头行驶, 车轮 与钢轨接触点位置发生较大改变使车轮型面磨耗分 布随之改变, 左右曲线占比不均的影响得到平衡, 从而能够很大程度上缓解车轮偏磨, 且磨耗分布较 未掉头时更为均匀。 $4 \times 10^{4} \mathrm{~km}$ 及以下掉头里程工况 下, 轮缘磨耗差和踏面磨耗差在第一次掉头时均不 
足 $0.2 \mathrm{~mm}$, 随运行里程增加左右侧车轮磨耗几乎一 致, 车轮型面磨耗分布和外形情况较为类似, 偏磨 现象基本消除。考虑到车辆实际运营维护, 频繁掉 头导致人力、物力以及成本的增加, 不易实现, 综 合仿真结果及分析, 本文制定了最佳掉头里程数为 $2 \times 10^{4} \sim 4 \times 10^{4} \mathrm{~km}$, 此时车轮偏磨问题得到解决且易 于实现。值得思考的一点是, 不同的运行总里程可 能导致有不同的最佳掉头策略, 本文仅针对总里程 为 $20 \times 10^{4} \mathrm{~km}$ 时提出参考建议, 且不同类型的车轮 偏磨问题解决策略需要另作讨论。

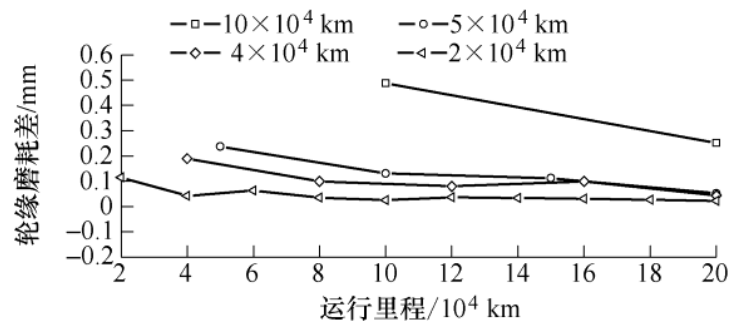

(a) 轮缘磨耗差

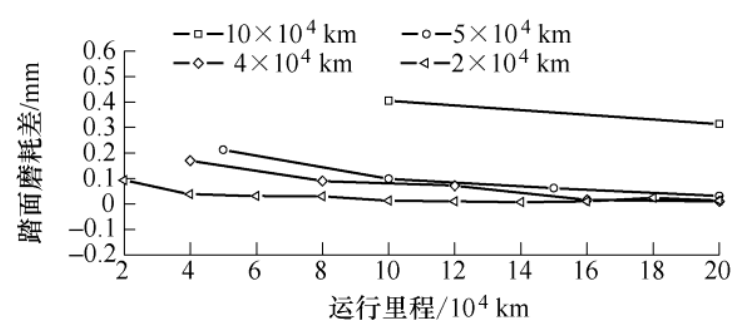

(b) 踏面磨耗差

图 9 磨耗差随里程变化情况

\section{2 小半径曲线占比对车轮磨耗的影响}

由车轮磨耗测试及数值仿真结果可知, 小半径 曲线的不对称分布是造成车轮偏磨的重要原因, 特 别是半径小于 $500 \mathrm{~m}$ 的曲线。为研究小半径曲线占 比对车轮磨耗的影响, 对本文的小半径曲线占比进 行适当调整, 在保证累积量不变的情况下, 改变左 右曲线的比例, 表 3 所示为小半径曲线占比相差 $1 \%$ 及 $2 \%$ 时比例设置情况。同时, 其余半径曲线及直 线段比例保持不变, 仿真计算过程及其他条件与前 文相同。

表 3 左右曲线不同占比

\begin{tabular}{cccc}
\hline 曲线半径 $/ \mathrm{m}$ & 左曲线 $(\%)$ & 右曲线 $(\%)$ & 累积 $(\%)$ \\
\hline \multirow{2}{*}{350} & 6.15 & 5.65 & \\
& 6.4 & 5.4 & 11.8 \\
\hline \multirow{2}{*}{450} & 9.115 & 8.615 & \multirow{2}{*}{29.5} \\
& 9.365 & 8.365 & \\
\hline
\end{tabular}

图 10 给出小半径曲线不同百分比下左右侧车 轮轮缘与踏面的磨耗差变化情况, 图 11 为左右曲线 比例相差 $1 \% 、 2 \%$ 及 $3 \%$ 时车轮型面磨耗分布及外 形。结果表明, 小半径曲线占比不均对车轮磨耗影
响较大, 车轮磨耗差随百分比差值的减小而减小, 印证了小半径曲线的不对称分布是造成车轮偏磨的 重要原因。该地铁线路半径小于 $500 \mathrm{~m}$ 的曲线中, 左右曲线比例相差达 $6.61 \%$, 左曲线长度几乎为右 曲线两倍之多，这是造成车轮偏磨的关键因素。当 百分比由 $6.61 \%$ 减至 5\% 时, 偏磨得到较大缓解, 轮 缘磨耗差下降 $21.4 \%$, 踏面磨耗差下降 $27.9 \%$, 百 分比由 $4 \%$ 减至 $3 \%$ 时, 磨耗差下降比较明显, 且此 时轮缘磨耗差与踏面磨耗差均不足 $0.5 \mathrm{~mm}$, 磨耗速 率差值小于 $0.025 \mathrm{~mm} / 10^{4} \mathrm{~km}$, 此时可不采取掉头措 施, 对今后地铁设计规划提供参考建议。从车轮磨 耗分布及外形可知, 右侧踏面磨耗依旧比左侧高, 这是因为整体线路左右曲线的不对称分布所导致。 若车辆运营不能做到经常掉头, 建议对小半径曲线 段采取不同的润滑措施, 在装置轨侧润滑设备时, 适当降低左侧车轮轮缘与钢轨接触的摩擦因数, 亦 或在车辆左侧加装轮缘润滑器, 以减轻车轮偏磨。

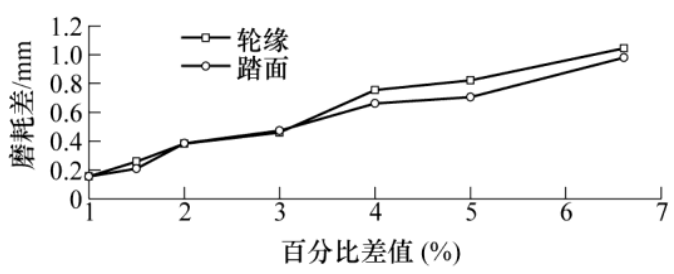

图 10 磨耗差随小半径曲线百分比差值变化情况

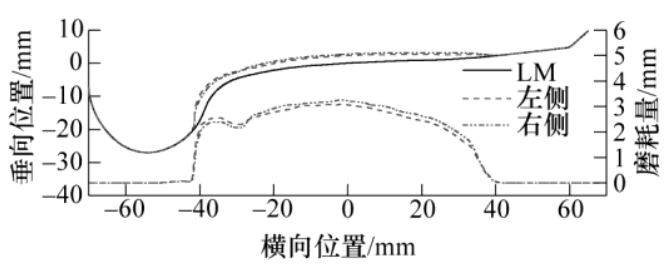

(a) 左右曲线占比相差 $1 \%$

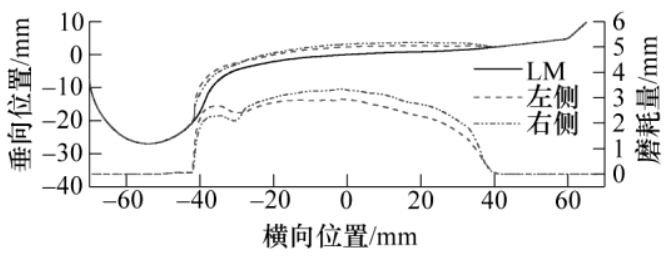

(b) 左右曲线占比相差 $2 \%$

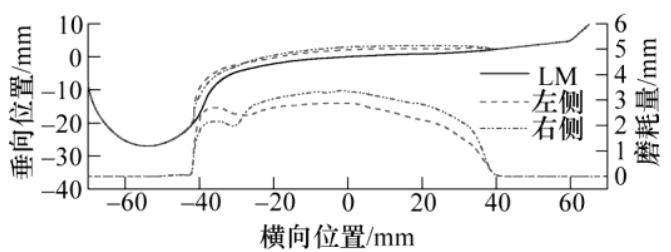

(c) 左右曲线占比相差 $3 \%$

图 11 小半径曲线不同占比车轮型面磨耗情况

\section{4 结论}

本文对某地铁线路的车轮磨耗进行了测试, 掌 握了磨耗规律。基于 UM 建立了车轮磨耗预测模型 
并进行验证, 分析了偏磨产生的原因, 提出了车轮 偏磨解决对策, 研究了掉头行驶以及小半径曲线占 比对车轮磨耗的影响, 制定了合适的掉头策略, 主 要结论如下。

(1) 对某地铁线路进行车轮磨耗测试, 发现所测 试的车轮存在严重的偏磨现象, 具体表现在左侧车 轮轮缘磨耗比右侧高, 右侧车轮踏面磨耗比左侧高。

(2) 基于 UM 建立了包括车辆动力学模型、轮 轨接触模型以及 Archard 材料摩擦磨损模型为一体 的车轮磨耗预测模型, 采取了合理的轨道线路统计 方法, 仿真与试验结果吻合较好, 车轮偏磨现象较 为明显。

(3) 分析了车轮偏磨产生的原因, 线路中小半 径曲线占比高、左右曲线严重不对称是主要原因, 车辆不掉头行驶使偏磨现象更加严重。定期掉头行 驶能缓解车轮偏磨现象, $4 \times 10^{4} \mathrm{~km}$ 及以下掉头时左 右侧车轮磨耗几乎一致, 最佳掉头里程数为 $2 \times 10^{4} \sim$ $4 \times 10^{4} \mathrm{~km}$ 。

(4) 小半径曲线占比对车轮磨耗有较大影响, 左右曲线百分比差值为 $3 \%$ 时轮缘磨耗差与踏面磨 耗差均不足 $0.5 \mathrm{~mm}$, 磨耗速率差值小于 0.025 $\mathrm{mm} / 10^{4} \mathrm{~km}$, 此时可不采取掉头措施。

本文研究方法和结果可为解决车轮偏磨问题和 地铁新线选线设计提供重要参考。

\section{参 考 文 献}

[1] 王开团, 张现锋, 周海廷, 等. CRH1 型动车组轮缘异 常磨耗研究及解决方案 [J]. 铁道机车车辆, 2019, 39(3): 114-117.

WANG Kaituan, ZHANG Xianfeng, ZHOU Haiting, et al. Research on abnormal wheel-flange wear of CRH1 EMU and its solutions[J]. Railway Locomotive \& Car, 2019, 39(3): 114-117.

[2] 齐万明, 慕光远, 胡建成. 哈尔滨地铁 1 号线车轮踏面 异常磨耗原因分析 [J]. 城市轨道交通研究, 2017, 20(11): 14-16, 89 .

QI Wanming, MU Guangyuan, HU Jiancheng. Analysis on abnormal wear of wheel tread on Harbin metro line 1[J]. Urban Mass Transit, 2017, 20(11): 14-16, 89.

[3] 尚小菲, 孟繁辉, 曲志及. 深圳地铁 2 号线车轮踏面异 常磨耗问题的原因分析及解决措施 [J]. 铁道机车车辆, 2019，39(2): 87-90.

SHANG Xiaofei, MENG Fanhui, QU Zhiji. Cause analysis and solutions for abnormal wear of wheel tread on Shenzhen metro line 2[J]. Railway Locomotive \& Car, 2019, 39(2): 87-90.

[4] 刘明珠. 重庆地铁一号线轮对异常磨耗问题及对策研 究[J]. 河南科技, 2018，44(11): 92-94.
LIU Mingzhu. Preliminary study on abnormal abrasion of wheelset on Chongqing metro line 1 and its countermeasure[J]. Henan Science and Technology, 2018，44(11): 92-94.

[5] 杨斌, 郭立昌, 郭俊, 等. 基于 $T \gamma / A$-磨损率模型的车 轮磨耗仿真分析 $[\mathrm{J}]$. 机械工程学报, 2017，53(22): 101-108

YANG Bin, GUO Lichang, GUO Jun, et al. Simulation analysis of wheel wear based on the model of $T \gamma / A$-wear rate [J]. Journal of Mechanical Engineering, 2017, 53(22): 101-108.

[6] 周素霞, 薛芯, 张寒, 等. 地铁线路曲线段磨耗状态下 轮轨滚动接触有限元分析[J]. 机械工程学报, 2016, 52(12): 129-135.

ZHOU Suxia, XUE Rui, ZHANG Qian, et al. Wheel-rail rolling contact finite element analysis of the wear state on the curve of subway line[J]. Journal of Mechanical Engineering, 2016, 52(12): 129-135.

[7] LI Xia, JIN Xuesong, WEN Zefeng, et al. A new integrated model to predict wheel profile evolution due to wear[J]. Wear, 2011, 271: 227-237.

[8] 李霞, 温泽峰, 金学松. 地铁车轮踏面异常磨耗原因分 析[J]. 机械工程学报，2010，46(16): 60-66.

LI Xia, WEN Zefeng, JIN Xuesong. Analysis of abnormal wear on metro wheel tread[J]. Journal of Mechanical Engineering, 2010, 46(16): 60-66.

[9] 李艳, 张卫华, 周文祥. 车轮型面磨耗对车辆服役性能 的影响 $[\mathrm{J}]$. 西南交通大学学报, 2010, 45(4): 549-554. LI Yan, ZHANG Weihua, ZHOU Wenxiang. Influence of wear of wheel profile on dynamic performance of EMU[J] Journal of Southwest Jiaotong University，2010，45(4): 549-554.

[10] 李艳, 张卫华, 池茂儒, 等. 车轮踏面外形及轮径差对 车辆动力学性能的影响 [J]. 铁道学报, 2010, 32(1): 104-108.

LI Yan, ZHANG Weihua, CHI Maoru, et al. Influence of wheel tread profile and rolling diameter difference on dynamic performance of vehicles[J]. Journal of the China Railway Society, 2010, 32(1): 104-108.

[11] 于春广, 陶功权. 地铁车轮磨耗测试及数值仿真 $[\mathrm{J}]$. 工 程力学，2016，33(1): 201-208.

YU Chunguang, TAO Gongquan. Analysis of metro wheel wear based on field measurement and numerical simulation[J]. Engineering Mechanics, 2016, 33(1): 201-208.

[12] FRÖHLING R D. Analysis of asymmetric wheel profile wear and its consequences[J]. Vehicle System Dynamics, 2006, 44: 590-600.

[13] 池茂儒, 张卫华, 曾京, 等. 轮径差对车辆系统稳定性 的影响 $[\mathrm{J}]$. 中国铁道科学, 2008, 29(6): 65-70.

CHI Maoru, ZHANG Weihua, ZENG Jing, et al. Influence 
of wheel diameter difference on the stability of vehicle system[J]. China Railway Science，2008， 29(6): 65-70.

[14] 黄照伟, 崔大宾, 杜星, 等. 车轮偏磨对高速列车直线 运行性能的影响 [J]. 铁道学报, 2013, 35(2)：14-20. HUANG Zhaowei, CUI Dabin, DU Xing, et al. Influence of deviated wear of wheel on performance of high-speed train running on straight tracks[J]. Journal of the China Railway Society, 2013, 35(2): 14-20.

[15] KARTTUNEN K, KABO E, EKBERG A. Numerical assessment of the influence of worn wheel tread geometry on rail and wheel deterioration[J]. Wear, 2014, 317: 77-91.

[16] 金学松, 刘启跃. 轮轨摩擦学[M]. 北京: 中国铁道出 版社, 2004.

JIN Xuesong, LIU Qiyue. Tribology of wheel and rail[M]. Beijing: China Railway Press, 2004.

[17] 罗仁, 石怀龙. 铁道车辆系统动力学及应用[M]. 成都:
西南交通大学出版社, 2018.

LUO Ren, SHI Huailong. Dynamics of railway vehicle systems and application[M]. Chengdu : Southwest Jiaotong University Press, 2018.

[18] PIOTROWSKI J , KIK W. A simplified model of wheel-rail contact mechanics for non-Hertzian problems and its application in rail vehicle dynamic simulations[J]. Vehicle System Dynamics， 2008， 46(1-2): 27-48.

[19] ARCHARD J F. Contact and rubbing of flat surfaces[J]. Journal of Applied Physics, 1953, 24(8): 981-988.

作者简介: 肖国放, 男, 1993 年出生。主要研究方向为铁道车辆车 轮磨耗。

E-mail: 13281187381@163.com 温泽峰(通信作者), 男, 1976 年出生, 博士, 研究员, 博士研究生导师。 主要研究方向为轮轨关系与减振降噪。

E-mail: zfwen@home.swjtu.edu.cn 